\title{
Implementing Digital Image Correlation for Determining the Tensile Characteristics of Post-Processed Thin Sheet Metal
}

\author{
Andrew D. Evans ${ }^{1, a}$, Amit Das $^{1}$ and Nick Croft ${ }^{1}$ \\ ${ }^{1}$ College of Engineering, Bay Campus, Swansea University, Fabian Way, Swansea, United Kingdom, SA1 8EN
}

\begin{abstract}
Acquiring material properties for finite element analysis is a necessity for producing accurate response outputs. In the sheet metal forming industry, there are many challenges in gaining material property definition from post-processed and/or extremely thin sheet metal. The difficulty in obtaining this information is the founding reason for applying digital image correlation in the characterisation process of post-processed beverage cans. These beverage cans have been cupped, drawn, re-drawn, ironed and stoved, so these can be described as "pre-necked" beverage cans. Due to the residual stress/strain worked into the material, it means that the specimens curl, hence, there are difficulties in attaching contact extensometers. This experiment has shown that the preparation and testing of test specimens is critical in obtaining accurate and robust results. The robustness has been fulfilled by ensuring the data matches contact extensometers, and the number of test specimens used is sufficient. This experiment has proven that the application of digital image correlation has produced reliable and useful tensile properties for the finite element model, whilst demonstrating the the thermal effects imposed during the stoving process, which in-turn has raised questions over the impact of thermal effects in the processing of cans.
\end{abstract}

\section{Introduction}

To manufacture a beverage can, there is a number of metal processing techniques required to manipulate the sheet metal into the cylindrical cup shape. These forming techniques include cupping, drawing, re-drawing, ironing, and necking.

In the "die necking" process used in the beverage can industry, the body is diametrically reduced to accommodate the joining of the beverage can end - which is financially beneficial to the process. This process is completed over a number of stages to reduce the likely hood of wrinkling.

The general physics of the "Die necking" process comprises of compression in the axial, circumferential and radial directions. In essence, this processing is exploiting the natural compressive instability of sheet metal.

Wrinkling is a phenomenon that is caused by compressive instability. This instability causes the metal to fold and collapse in a manner similar to pleating. This failure can cause the can to be rejected from the line, cause blocking of the equipment, increased tool wear and financial implications on the process.

There is huge research investment into reducing wrinkling effects in the "die necking" process, utilising numerical tools such as finite element analysis (FEA).

FEA is a tool that requires the user to accurately input the physics, geometry and material characteristics of the problem to gain desired outputs, such as stress, strain, forces etc. The inputted values are important due

\footnotetext{
a Corresponding author: 487056@swansea.ac.uk
}

to the fact that the reliability and accuracy of the output is heavily reliant on the input parameters i.e. garbage-in, garbage-out (GIGO).

Due to the number of stages used prior to the "die necking" phase, re-characterisation of the material properties is required to ensure accuracy and reliability of the model outputs. It is generally well known that FEA has a corresponding error, this will be magnified over a number of simulations. The magnitude of this error will naturally limit the number of simulations that can take place.

Re-characterising pre-processed material poses huge obstacles caused by the thin nature of the metal $(0.155$ $\mathrm{mm})$, the curved nature of the cup and the residual stress in the metal.

\section{Process definition}

The process of necking is relatively straight forward to understand. It is important to fully comprehend the geometric setup and material characteristics of the can body - in addition to the dynamics of the process.

It is common to expect the necking process to produce approximately 3.5 cans every second. At this rate, the loads on the beverage can are highly significant to the structural integrity of the beverage can bodies and the robustness/reliability of the process. 


\subsection{Body wall geometry}

As shown in Figure 1, the cross-section of the body wall will show that the wall has no uniform thickness distribution. The thickness distribution in the axial direction will show that the top wall thickness, mid wall thickness and bottom wall thickness all differ. This difference has been applied for a purpose.

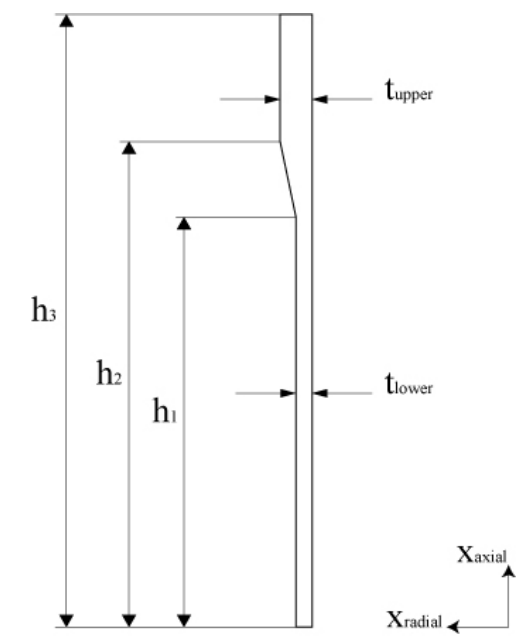

Figure 1. Demonstrating the thickness distribution of the beverage can in the axial direction.

If a cross-section of the body wall is taken on the radial - circumferential plane, there will be a nonuniform distribution in the thickness. This distribution is much harder to measure as the magnitude is approximately $10^{-3} \mathrm{~mm}$; however, the existence of this thickness distribution can be confirmed using the poisons ratio relations when measuring the wall height of the can as shown in Figure 2. During the drawing/ironing processes, the observing party will notice a considerable change in the axial wall height. This correlates directly the the anisotropic nature of the aluminium material [1].

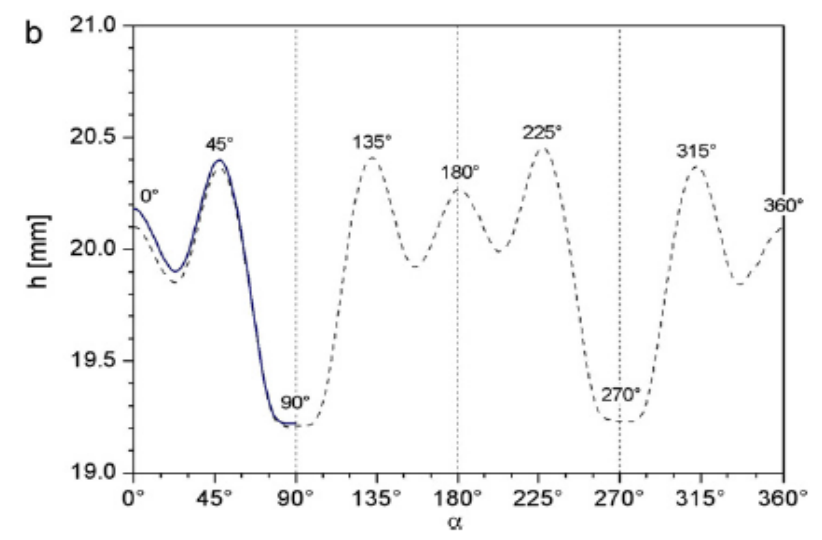

Figure 2. Showing the trend of the earing profile of the beverage height caused by anisotropy [1].

\subsection{The "Die Necking" process}

The die necking process usually comprises of multiple manufacturing stages. Each stage will gradually reduce the diameter of the body ready for the attachment of the lid. Generally, the process is identified as a "bottle neck" in the process, in-terms of speed, cost and reliability. For these reason, there is increased research investment in the optimisation of the process.
(1)

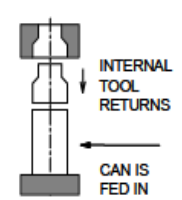

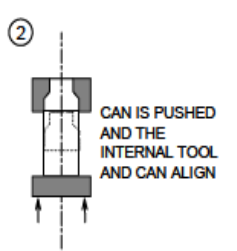

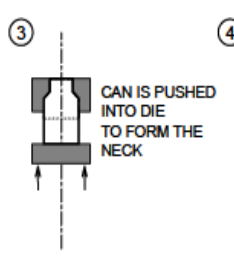

(4)

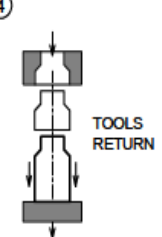

Figure 3. Showing the general forming operation of the beverage can in the "die necking" stage [2].

The tooling for the process requires a die, knockout and pusher/feeder. These tools work in tandem to conduct the forming operation required. The die is fully constrained, and the feeder and the knockout share the same constraints where UR1 $=\mathrm{UR} 2=\mathrm{UR} 3=\mathrm{U} 1=\mathrm{U} 2=$ 0 . U3 is free to move and represents the axial direction.

As shown in Figure 3, the process operates in four main stages, where:

- Stage 1 - in this part, the can body is displaced towards the die by the pusher/feeder working in the U3 (axial) direction.

- Stage 2 - in this part the body and the die make contact, forcing the can body to be relatively concentric. Once the seal is airtight between the top edge of the can body and the wall of the die, internal air pressure is increased.

- Stage 3 - the body wall makes contact with the die, and subsequently follows the profile of the tooling. During this forming stage, the can wall deflects off the knockout during contact.

- $\quad$ Stage 4 - The internal air pressure is used to force the can off the die, once forming is complete, and the can body is moved to the next staged.

\subsection{Pre-processed material data}

Characterising the material is vital for fully understanding and defining the mechanical limits for manufacture. With this in mind, a fully explored materials characterisation program must be introduced. The pre-processed material properties have been defined using standard contact tensile testing for the materials characterisation, and provides a benchmark for further investigations. This is shown in Figure 4, where the engineering stress and strain is plotted.

The pre-processed material tensile data shown in Figure 4, must be compared with the post-processed material data to fully understand the changes of the material between the pre-processed and pre-necking stages.

The pre-processed material data demonstrates that there is an anisotropic relationship present within the material. 


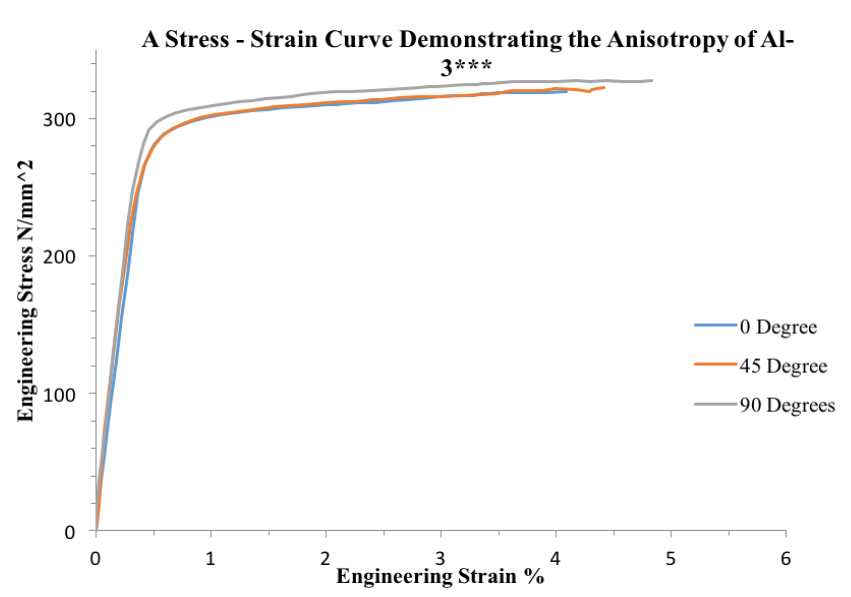

Figure 4. Showing the trend of the engineering stress-strain curve for pre-processed aluminium $3 * * *$ series.

\section{Characterisation requirements}

In order to produce a material input card that can provide an accurate representation of the state of the material, certain test parameters need to be chosen to define the experiment. These parameters/experiments are:

- Top wall characterisation must be used for the material input card. The top wall is where the material will be necked, therefore a greater load and deformation will occur in this region - making it critical for robustness and accuracy.

- Cauchy Stress/Strain data including the plastic and elastic regions of the curve, UTS (ultimate tensile strength), yield stress and maximum strain - This is the basic material data that is required for any material characterisation.

- Comparison of multiple specimens and mean data profile - a comparison of multiple specimens will produce a quantifiable representation of the extreme results. The mean data will be required for the majority of simulations.

- Comparison between pre-processed, and pre-necked material - it will be interesting to show the change in profile of the material once it has been processed.

- Comparison between stoved and non-stoved material to quantify the difference (if any), that may exist - stoved can body stock may have had some release in residual stress/strain, which will incur some changes to the material properties.

- Anisotropic material data - it is vital to build a data bank that will include anisotropic data. Anisotropy is an inherent property in sheet metals, so it is important to capture this behaviour.

\section{Characterisation options}

Characterising thin sheet metal is very problematic, especially when mechanic data such as stress-strain data is required. Largely, the main challenges faced with thin sheet metal is witnessed in the use of contact extensometers.
Before a relevant method for characterisation can be selected for the experiment, there must be a sufficient exploration into the viable options available for the experiment.

\subsection{Extensometers}

There are two main types of extensometers under consideration, which include contact, and non-contact extensometers.

\subsubsection{Contact Extensometers}

These are a more traditional type of extensometer which is designed for most routine applications where surface normal forces may not be an issue, high energy events are not used and damage from the extensometer being contact with the test specimen is not an issue. Contact extensometers are available in generally two variations, clip-on and sensor arms.

The clip-on variation is an individual unit that can be fixed onto the surface of the specimen. This is suitable for most applications; however, when applied to very thin samples below $1 \mathrm{~mm}$ in this instance, it is expected that the extensometer will produce a surface normal force, and may cause inaccuracies in the result. During initial testing, the clip-on extensometers proved to be inaccurate due to slippage of the contact feet. This caused the data to be unusable for any practical uses.

The sensor arm type of extensometer consists of two prongs that are placed at the top and bottom of the gauge of the specimen. The prongs are fixed to the edge of the sample rather than the surface to reduce any influence on the data. In some instances, slippage of the arms can be witnessed due to natural bends in the test specimens caused by residual stresses.

\subsubsection{Non-Contact Extensometers}

Non-contact extensometers are usually applied to scenarios where explosive failure or high strain-rates where specimens maybe influenced by surface forces or non-standard geometries may exist. In-terms of accuracy, non-contact extensometers can be better in high strainrates. Non-contact extensometers are usually more expensive than contact extensometers.

In this project, due to availability, the non-contact extensometers under consideration are laser and videobased extensometers such as digital image correlation.

Laser extensometers can provide a good alternative to standard contact methods. They have the capability of measuring strain to a similar standard, but with the advantage of being able to operate in high temperatures. These operate by projecting a grid pattern onto the surface of the specimen and tracking the deformation. More modern systems can be integrated into the tensometer rigs and provide very accurate results in the micro and macro scale.

Video extensometers are available in different forms, but the type of most interest to the project is called Digital Image Correlation (DIC). DIC uses two cameras 
to record images at high frame rates of 5,400 at a 1million-pixel density. The specimen must be coated in a matt based paint with maximum contrast. In other words, the specimen must have a white speckled pattern on a black background or vice versa. The DIC software is programmed to track unique patterns, so there is no need for a repetitive pattern being applied. DIC is a great alternative to laser extensometers, and can provide more data through its software. It is suitable for strains greater than $0.01 \%$. DIC is expensive to buy with a cost of $£ 150,000$ per unit; however, the equipment is available for free rental through the EPSRC Loan pool [3].

\subsection{Extensometers conclusion}

Due to the restrictions imposed by specimen curvature caused by residual stress, and extremely thin aluminium specimens, it seems only reasonable to implement a noncontact extensometer. With this in mind, the use of a video based extensometer or laser may prove to be beneficial. Due to issues obtaining laser extensometers, the only option available is to use digital image correlation equipment provided by the EPSRC loan pool.

\section{Experimental Apparatus, Setup and Sample Preparation}

Using DIC requires an investment in time to setup the equipment correctly. The majority of the setup is simple to conduct, and is a case of connecting wires. There are some portions of the setup that are very important, which may produce errors if completed poorly. Usually the ideal setup cannot be arranged due to space and movement limitations, and this setup is no different. As shown in Figure 5, the setup of the cameras is positioned either side of the pillars.

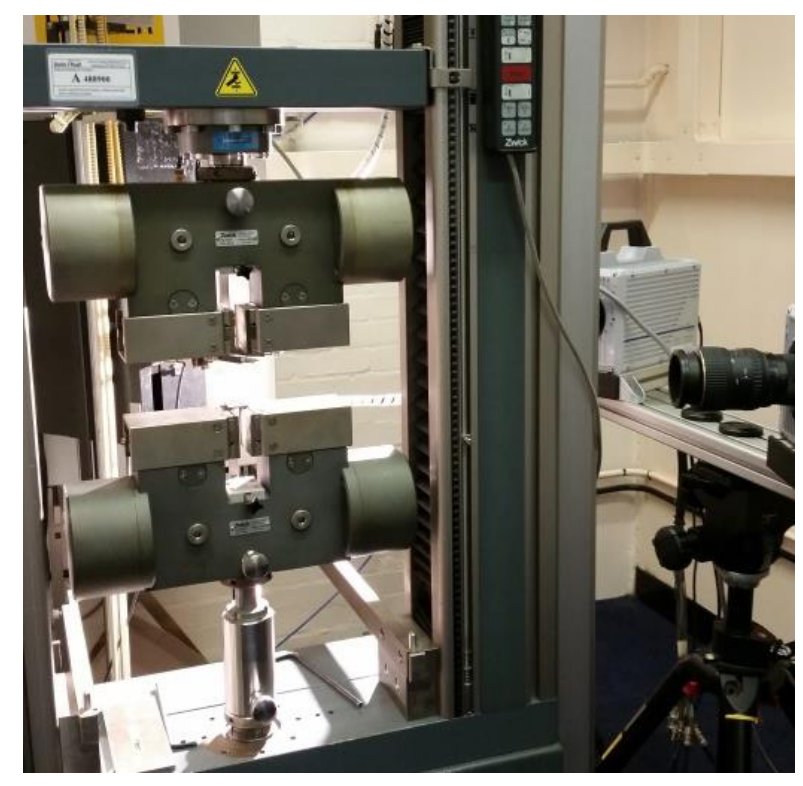

Figure 5. showing the general setup of the cameras. :- note the placement of the pillar and cameras.

\subsection{Apparatus}

The DIC equipment is supplied through the EPSRC loan pool at no cost to the project. The equipment supplied is:

- $2 \mathrm{x}$ Photron SA1 Camera Processors - This is the two high speed cameras shooting at $1 \mathrm{MP}$ and up to 5,400 frames per second (fps).

- Adaptor rings for lenses - These are required for different mounting rings for different brands of lenses.

- Cable remote shutter cable - This is used to start/stop the recording of the camera.

- Tokina $100 \mathrm{~mm}$ fl2.8 macro lens - There is a choice of different lenses, but due to the size of the specimens, a larger focal length with a smaller focus distance is required.

- Heavy duty tripod and camera mounts - The tripod must be adequate to withstand the mass of two cameras. The cameras must be mounted at the same height, so the large camera mount is used.

- Continuous lighting with barn door attachments - The lighting is used in poorer lighting conditions where the light may not be stable or intense enough. Higher f-stops may be required, so more light will be required to illuminate the subject.

- Calibration grids - Calibration grids are required for the setup of the software.

- VIC-3D Software - This conducts the analysis, and is used to extract the strain data from the experiment.

\subsection{Apparatus Setup}

The initial setup of the DIC equipment is important to ensure that there is minimal opportunity for discrepancies to influence the result.

\subsubsection{Camera Position}

Firstly, the DIC equipment needs to be setup correctly. As shown in Figure 6, the angle between the two cameras and the specimen must be between $15^{\circ}$ and $60^{\circ}$ ensuring that the cameras are symmetric relative to the specimen.

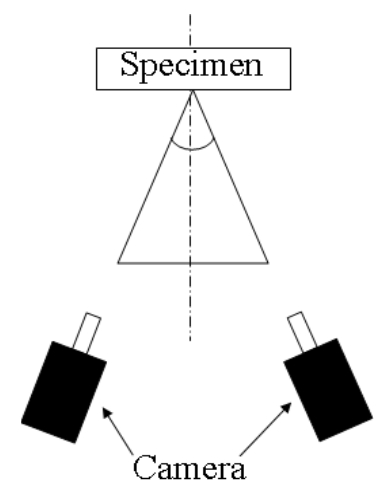

Figure 6. showing the general setup of the two cameras and the specimen, with particular focus of the angle and symmetry between the two cameras. 


\subsubsection{Lens Selection}

In this case, there is a choice between a $100 \mathrm{~mm}$ macro and a $24-85 \mathrm{~mm}$ lens. The choice of lens is dependant on the size of the specimen, and the distance between the camera and the specimen. Due to the large distance required between the specimen and the camera, caused by the pillar of the tensometer obscuring the line of sight, the larger $100 \mathrm{~mm}$ lens is required.

\subsection{Camera settings}

At this point, the calibration grid can be placed into the region where the tensile specimen will be loaded for the correct exposure. The exposure can be controlled with a number of different settings on the camera or by using controlled lighting. This is similar to standard photography, where there is usually a balance between a number of factors in-order to gain the correct exposure. In this particular scenario, the exposure was controlled by frame rates, aperture and continuous lighting. camera and the specimen.

To achieve the correct output data, the user must prioritise the exposure factors:

1. Frame-rates - this is usually measure in frames per second (fps), and is the primary factor for the data output. Due to the tensometer having an output of 50 data points per second, the cameras must be set to this number, or a divisional of the tensometer data output. A scenario where the fps must be set as a divisional value is if the aperture of the lighting reaches its upper limit. In the experiment, the frame-rate is set to $250 \mathrm{fps}$, with the intention of saving 1 in 5 frames as an output.

2. Aperture - The aperture of the camera is determined by the amount of light the lens can allow to enter the camera sensor. A lower f number (i.e. f2.8) will let in more light than a higher f number (i.e. f22). Also, the lower $f$ number will have a much greater depth of field than that the higher value, which means that more of the background will be softer/out of focus in comparison to the high $\mathrm{f}$ number. In this experiment, the tensile specimen must be in focus, and due to a relatively close focus distance, the depth of field is exaggerated, so, f16 is used. This is also the highest number that is run on the experiment, even though the maximum is $\mathrm{f} 22$. This is due to lens imperfections such as dust or scratches, become visible over f16, and may skew the results.

3. Continuous lighting - fortunately, the testing laboratory chosen for the tensile testing has low ambient light, which means that the light output can be controlled with a much greater accuracy. The lighting is controlled using a swivel switch, but there is no measurement output of the light being emitted. Therefore, this is controlled by the user. The light output is strong, and even on a matt white surface, some reflections occur. Therefore, it is recommended to take the light output to the highest possible light output with no reflections visible on screen.

\subsection{Sample preparation}

The accuracy of the output data produced by the DIC system is partly reliant on the preparation of the tensile specimen. The specimen dimensions are shown in Figure 7.

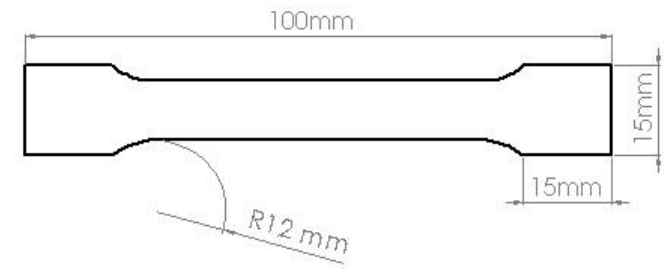

Figure 7. Showing the schematic of the tensile specimen used in the DIC testing.

The specimen chosen in Figure 7 was selected due to the size being perfectly suited to the dimensions of the can body. The can body was required to be altered slightly to accommodate any specimen. This alteration extends the top-wall thickness down the entire wall of the can. The top wall thickness is required for testing because it is the region that is necked during the process. The extension of the top-wall thickness is completed using protrusion dies, which will produce the same thickness, and the (approximate) same strain as the topwall. This profile is demonstrated in Figure 8.
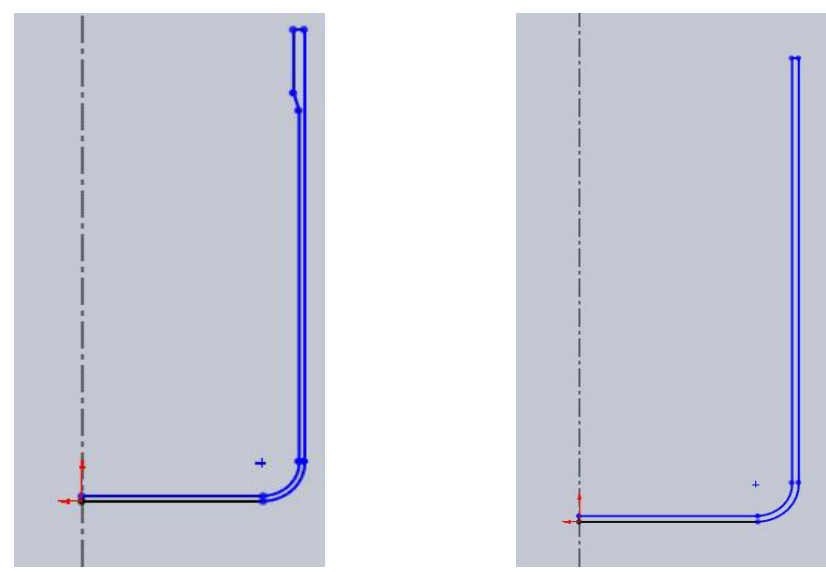

Figure 8. (Left) showing the Original can cross-sectional thickness profile and (Right) showing the can protrusion of the cross-sectional thickness.

Once the test specimens have been punched and the thickness measurements have been recorded, the paint must be applied. This step is crucial for producing accurate results, and the requirements include having a high contrast speckled pattern applied to the specimen [4].

For maximum contrast, it is recommended that the tester uses either white speckles on a black undercoat, or visa versa. The speckles must not be applied using a specific uniform pattern, they must be random. This random pattern ensures that the software can identify a unique area of the specimen and track it for the duration of the trial. The paint should be applied with reference to Figure 9 [4]. 


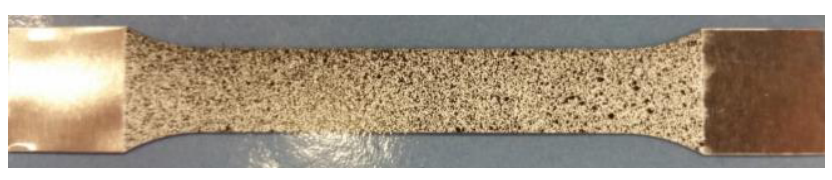

Figure 9. Illustrating the desired speckle pattern.

\subsection{Experiment sample size}

To ensure that the experiment is conducted to the required standard, a variety of different experiments will take place. The main focus of this program is to produce a material input card, with a secondary focus on the thermal effects that the stoving process may have on the metal. With this in mind, three tests were constructed:

1. Benchmarking - the benchmark test is used to compare standard pre-processed material using a standard arm extensometer for direct comparison with the digital image correlation strain data. This will ensure that the test data is reliable. In this test, 10 specimens will be used for each extensometer.

2. Heat treatment comparison - This will compare the data produced from stoved can bodies with nonstoved bodies. This will highlight whether there is any release in residual stress/strain.

3. Anisotropy - There will be a comparison between the axial and circumferential specimens to characterise the anisotropic nature of the material.

4. For the remaining experiments, 40 test specimens will be used for stoved and unstoved with a total of 80 for both scenarios. These will have an equal proportion of 20 axial and circumferential specimens for both.

\section{Results}

As discussed in the previous Section 5.3, there will be a total of 3 experiments to ensure that the test has been conducted thoroughly.

\subsection{Benchmarking - DIC comparison with contact extensometer}

As shown in Figure 10, both curves are closely matched in the plastic region of the graph with some difference in the elastic region. At the start of the DIC curve at approximately $10 \mathrm{~N}$, there us a slight change in gradient before the curve becomes linear until Yield. There is very little difference between the gradient between the two curves otherwise.

The yield point for both methods is at $260 \mathrm{MPa}$ and the Ultimate Tensile Strength (UTS) is measure at 216 $\mathrm{MPa}$ for both. The maximum Engineering Strain is measured at $6.2 \%$ and $6.03 \%$ for DIC and Contact Extensometer respectively.

There are important observations to understand when using DIC, which may have a noticeable impact on the results. In these circumstances, this data can be disregarded. Such discrepancies include the lighting conditions, paint quality and camera knocks.
Overall, the reliability of DIC when compared to standard contact extensometers has proven to shown negligible discrepancies when observing the results shown in Figure 10.

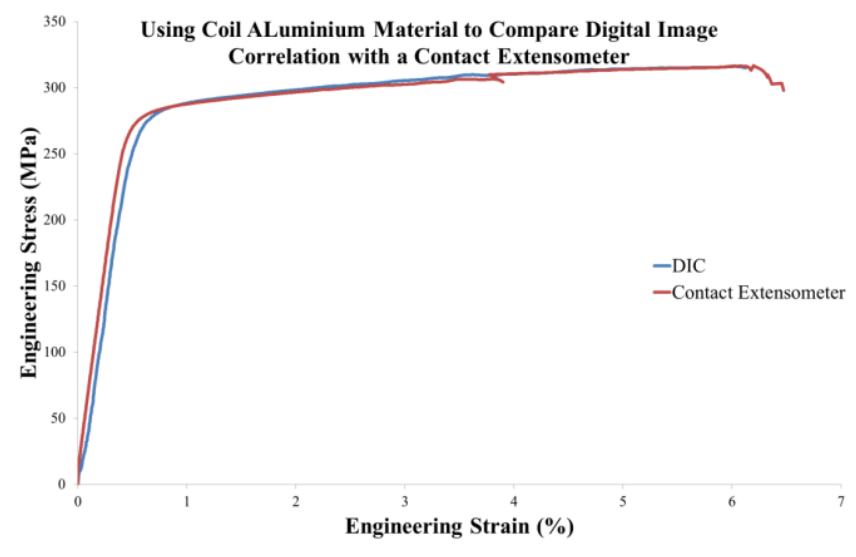

Figure 10. Comparing DIC with the Zwick contact extensometer. Stress/Strain Data Comparing Anisotropy Between Baked and Non - Baked
Body Stock.

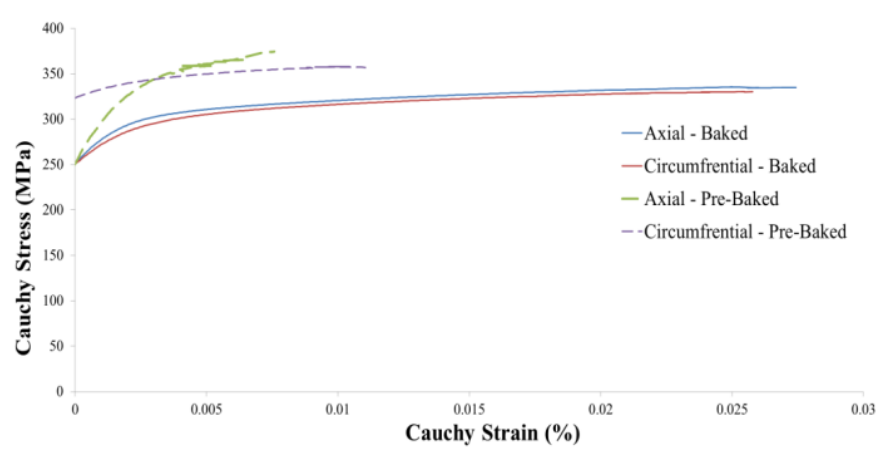

Figure 11. Showing the input Cauchy stress/strain data past the yield point for pre-processed (dashed lines) and post-processes (solid lines) material data for the circumferential and axial orientations. This is the format that Abaqus requires for its material input card definition.

\subsection{Anisotropy - Axial vs Circumferential}

As shown in Figure 11, the pre-baked material data (dashed lines) shows a distinct anisotropic trend after the yield point. The material yield occurs at $320 \mathrm{MPa}$ for the circumferential and $250 \mathrm{MPa}$ for the axial orientations.

The pre-baked material data for the axial specimens continues on a steeper gradient - when compared to the circumferential specimen - before failing at 0.065 strain and $375 \mathrm{MPa}$ stress.

The pre-baked material data for the circumferential specimens has a shallower gradient, when compared to the axial specimen. The failure of the circumferential specimen occurs at 0.012 strain at $350 \mathrm{MPa}$.

The post-baked material data for the circumferential and axial specimens follows similar trends, where the yield point is witnessed at $250 \mathrm{MPa}$, and the maximum stress is $320 \mathrm{MPa}$. The maximum plastic Cauchy strain is shown to be 0.025 for the circumferential and 0.027 for the axial specimens. 


\subsection{Comparing heat treatment}

As shown in Figure 11, there is a significant difference between the heat treated and non-heat treated samples. There is a minimum gain in strain of 0.015 and a maximum of 0.0175 when comparing axial samples and circumferential. There is also a significant decrease in stress of $50 \mathrm{MPa}$ for the circumferential samples and 65 MPa difference for the axial samples.

\section{Conclusions and perspectives}

The digital image correlation experiment has provided new means for testing materials where test specimens have a thin profile.

There are difficulties associated with the use of digital image correlation that need to be noted, which include, paint quality, lighting and compiling the strain data with the stress data. In other words, there is no real integrated approach for data aggregation.

The reliability and comparability of the DIC data with a proven contact extensometer has proven to be consistent. Over a test batch of 10 specimens per extensometer, there has been very good consistency, which reduces/eliminates elements of doubt.

The anisotropic tendencies found in the sheet metal post-baking and pre-baking, has shown that there is a significant change in the characteristics of the material to significant proportions. This change needs to be verified by observing the microstructure of the material; however, it is unlikely any phase transformations have occurred, and it is more likely that the changes have been merely releases of residuals.

Overall, the use of DIC has given an incite into the manufacturing process that has never been looked into in the past; which has subsequently opened new questions to be answered in-terms of the application of DIC in other processes and research topics into other stages. DIC has been important in providing an FEA model up-to-date and reliable data to conduct simulations, which will inevitably reduce the tendencies for errors to occur. This paper has highlighted the importance of materials characterisation for FEA and its unexpected influences on other projects.

This project has covered many aspects of the materials characterisation of thin sheet metals, and the challenges that are part of it; However, there hare some questions that have become visible due to the results of the experiment.

An investigation into the cause of the changes seen before and after stoving. This can be completed by viewing the microstructure, and searching for phase transformations.

It would be useful to conduct experiments using high strain rates. DIC will be able to be implemented for this once a suitable high strain rate tensometer can be located.

\section{References}

1. O. Engler, N. Mertens, P. van Dam, J. of Mat. Proc. Tech, 211 (2011), pp. $1278-1284$.
2. J.A. Jordan-Cordera, $8^{\text {th }}$ International LS-DYNA Users Conference, pp. 37-46 (2004).

3. S. Amini, R. Kumar, Mat. Sci. \& Eng, Structural Materials: Properties, Microstructures, and Processing, 594 (2014), pp. 394-403

4. V.P. Rajan, M.N. Rossol, F.W. Zok, Exp. Mech., 52 (2011), pp. 1355-1373 